\title{
The upgraded cold neutron triple-axis spectrometer FLEXX - enhanced capabilities by new instrumental options
}

\author{
Klaus Habicht ${ }^{1, \text { a }}$, Diana Lucía Quintero-Castro ${ }^{1}$, Rasmus Toft-Petersen ${ }^{1}$, Mathias Kure ${ }^{1,2}$, Lucas Mäde ${ }^{1,3}$, \\ Felix Groitl ${ }^{4,5}$ and Manh Duc Le ${ }^{6}$ \\ ${ }^{1}$ Helmholtz-Zentrum Berlin für Materialien und Energie GmbH, 14109 Berlin, Germany \\ ${ }^{2}$ Niels Bohr Institute, University of Copenhagen, 2100 Copenhagen, Denmark \\ ${ }^{3}$ Institut für Physik und Astronomie, Universität Potsdam, 14476 Potsdam, Germany \\ ${ }^{4}$ École Polytechnique Fédérale de Lausanne, Laboratory for Quantum Magnetism, 1015 Lausanne, Switzerland \\ ${ }^{5}$ Paul Scherrer Institute, Laboratory for Neutron Scattering, 5232 Villigen, Switzerland \\ ${ }^{6}$ Center for Correlated Electron Systems, IBS, Seoul National University, Seoul 151-747, Korea
}

\begin{abstract}
The upgrade of the cold neutron triple axis spectrometer FLEXX, a work-horse instrument for inelastic neutron scattering matching the sample environment capabilities at Helmholtz-Zentrum Berlin, has been successfully accomplished. Experiments confirmed an order of magnitude gain in flux now allowing for intensity demanding options to be fully exploited at FLEXX. In this article, we describe the layout and design of two newly available FLEXX instrument options in detail. The new Heusler analyzer gives an increase of the detected polarized neutron flux due to its superior focusing properties, significantly improving the feasibility of future polarized and neutron resonance spin echo experiments. The MultiFLEXX option provides simultaneous access to large regions in wavevector and energy space for inelastic excitations thus adding mapping capabilities to the spectrometer.
\end{abstract}

\section{Introduction}

The BER II neutron source and its instruments have recently undergone major developments. An extensive upgrade program was conducted which included the exchange of the cold source moderator cell while the complete guide system in the Neutron Guide Hall I was rebuilt with state-of-the-art supermirror guides [1]. Simultaneously, the cold neutron triple axis spectrometer FLEX [2], a work-horse instrument for inelastic neutron scattering matching the low temperatures and high magnetic fields sample environment capabilities at HZB, was upgraded and relocated to a guide end position [3]. Experiments performed during the commissioning phase confirmed an order of magnitude gain in intensity as a result of the upgrade [4].

In synergy with the extreme sample environment available at the HZB, the current gain in intensity allows for very demanding experiments to be conducted at FLEXX. It also allows for new instrument features, further enhancing the FLEXX capabilities. Neutron scattering experiments with longitudinal XYZ polarization analysis or the neutron resonance spin-echo (NRSE) option are examples of such intensity demanding modes in which FLEXX can be operated. To further enhance polarized neutron capabilities at FLEXX a new Heusler analyzer has been commissioned and is described here. Since the new primary spectrometer results in an increased flux at the sample position it is furthermore possible

\footnotetext{
a e-mail: habicht@helmholtz-berlin.de
}

to analyze the scattered neutrons in a broad range in wavevector and energy $(\mathrm{Q}, \mathrm{E})$ space. In this article we describe the basic design features of MultiFLEXX, a new optionally available secondary spectrometer. MultiFLEXX is designed for detecting neutrons in 31 different wavevector channels each with 5 different energy channels. This adds up to 155 points in (Q,E) space being simultaneously probed by this optional back-end.

This article is organized as follows: We first summarize the main new features of the upgraded FLEXX spectrometer and the comparison of its performance before and after the upgrade. In the Sect. 3 we focus on the properties of the new FLEXX Heusler analyzer. The design principles of the MultiFLEXX option are described in Sect. 4.

\section{FLEXX primary spectrometer upgrade}

FLEXX was relocated to a guide-end position in an area of the guide hall with intrinsically low background. The primary spectrometer of FLEXX was completely rebuilt with new $\mathrm{m}=3$ guides including a converging elliptical section with a supermirror coating gradually increasing from $m=3$ to $m=5$ to focus neutrons onto a vertical virtual source $[5,6]$. The neutrons are subsequently imaged onto the sample position by a new double focusing monochromator, ensuring a drastic increase in neutron flux reaching the sample. Although this rise is at the expense of coarser Q-resolution, the beam monochromatization could be further increased by satisfying Rowland geometry focusing conditions [7]. In addition, a new velocity selector

This is an Open Access article distributed under the terms of the Creative Commons Attribution License 4.0, which permits unrestricted use, distribution, and reproduction in any medium, provided the original work is properly cited. 
is placed far upstream in the primary beam path. This greatly reduces the inelastic background and removes higher order scattering thus eliminating the need for filters.

The intensity at the sample position was determined in a series of monochromator rocking scans with a neutron monitor at the sample position for a range of monochromator scattering angles. The absolute flux was obtained from gold foil activation which is now routinely used for monitoring the guide quality. Experiments using a vanadium standard and the well-known phonon dispersion from a single crystalline specimen of elemental $\mathrm{Pb}$ confirmed an order of magnitude gain in intensity, and demonstrated that the incoherent elastic energy widths are measurably narrower than before the upgrade. The drastically improved count rate allows spectroscopy on smaller samples when operating the FLEXX spectrometer in standard triple axis mode and most notably allows using the so-called Dy-booster for inelastic measurements, which adds $2.5 \mathrm{~T}$ to the vertical field of the $14.5 \mathrm{~T}$ magnets but severely restricts sample volume. The improved spectrometer performance now allows for intensity demanding setups, such as XYZ polarization analysis, NRSE and the MultiFLEXX back-end.

The NRSE option available at FLEXX has been rebuilt along with the upgrade program [8]. The larger solid angle subtended by the double focusing monochromator required an adaptation of central components of the NRSE apparatus. New coupling coils and rebuilt bootstrap coils now provide a matched beam transmission while higher coil tilt angles can be accessed. The latter enables high energy resolution measurements on steeper dispersions. With its new spectrometer arms the instrument is more compact than before, enabling direct beam calibration measurements necessary for proper data reduction of Q-dependent linewidth measurements over the entire accessible wavevector range. In addition, the accessible Q-range for the NRSE option operated in Larmor diffraction geometry is increased by a factor of 2 . The polarized neutron flux at the sample position was increased by a factor of 5 . The use of the recently commissioned new Heusler analyzer results in a total gain factor of 15.5 in intensity for the upgraded NRSE option. The commissioning of the instrument demonstrated the reliable performance of the new NRSE option, now offered to users at FLEXX.

\section{FLEXX polarized option}

To complement the normal mode of operation of FLEXX, a polarization option is also offered. For this option a compact S-bender polarizer (see Fig. 1) mounted on a vertical guide changer may be translated into the beam at the end of the straight guide section, before the focusing elliptical guide. Its position in a region of the neutron beam path with lower divergence maximizes the polarized neutron flux at the sample position. The transmission profile of the S-bender is matched to the divergence transported by the neutron guide since the angular width of the polarizer transmission curve corresponds to the divergence of the $m=3$ supermirror guide. The transmission properties of the polarizer have been measured with rocking scans of the whole device

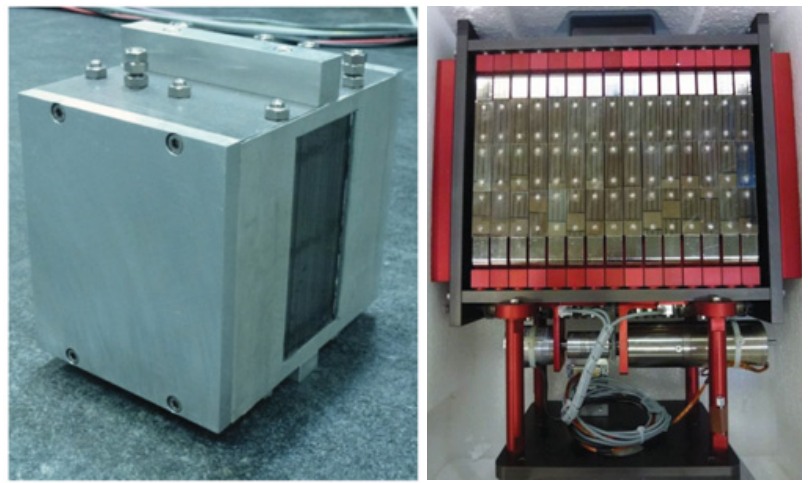

Figure 1. (Left) photography of the S-Bender used at FLEXX to polarize the incoming neutron beam built by Mirrotron Ltda. (Right) photograph of the Heusler analyzer used at FLEXX to analyze the scattered neutron beam.

and confirmed by transmission measurements at FLEXX comparing the total transmitted intensities with and without polarizer in the primary beam path.

Since the polarizer is mounted $5 \mathrm{~m}$ from the monochromator, permanent magnet guide fields are installed along the elliptical and vertical guides between the polarizer and monochromator in order to ensure the transmission of the neutron beam without loss of polarization.

A Heusler analyzer, with fixed vertical and variable horizontal focusing, can substitute the normally used pyrolitic graphite (PG) analyzer. The Heusler analyzer accepts only neutrons with vertical polarization and selects the final energy of the neutrons scattered from the sample.

The analyzer is made of 3 rows of 15 Heusler crystals of $\mathrm{Cu}_{2} \mathrm{MnAl}$ aligned along the $(1,1,1)$ Bragg peak in reflection geometry. The average crystal mosaicity of the individual Heusler crystals is $0.42^{\circ}$. The analyzer mechanics have been designed by SwissNeutronics and the Heusler crystals have been provided by the neutron optics group of the ILL (see Fig. 1). The essential advantage of this design is that each column of the analyzer is magnetized by its individual yoke. The yokes are made of $\mathrm{Nd}_{2} \mathrm{Fe}_{14} \mathrm{~B}$ magnets and soft iron, and are constructed such that no zero field regions exist before the analyzer. The field direction is vertical and points in the same direction as the projection of the magnetic field of the earth on a vertical axis. The magnitude of the magnetization field is calculated to be $0.17 \mathrm{~T}$ directly on the analyzer crystal surface.

First measurements have been performed in order to characterize the different polarization components. Measurements of the incoherent line of a vanadium rod have been made by setting the spectrometer in diffraction mode with $k_{i}=k_{f}=1.55 \AA^{-1}$ in $W$ configuration. Analyzer rocking scans have been performed for an unpolarized incident beam, for two different settings, using the PG analyzer and using the Heusler analyzer. Figure 2 shows the comparison between the two analyzer rocking scans with both analyzers operated in focusing mode. Note that an unpolarized neutron beam was used, and the intensity obtained by using the Heusler analyzer would be expected to be half of the intensity obtained by using the 


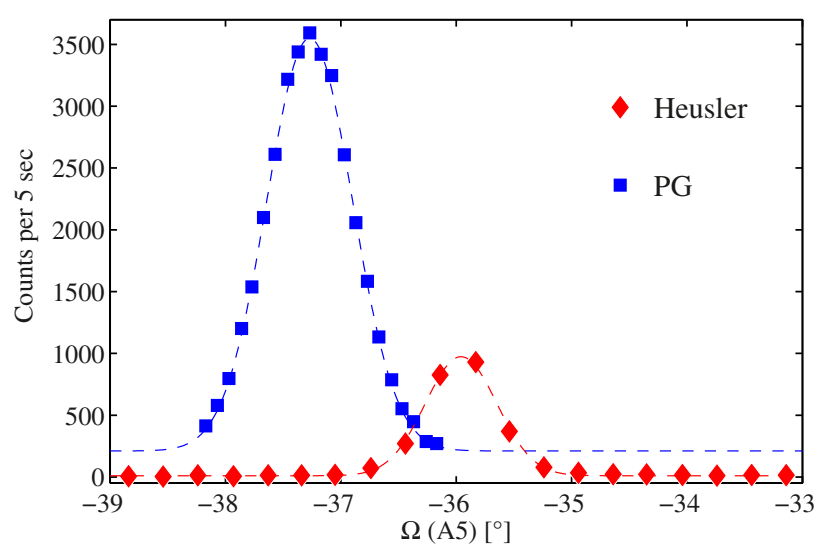

Figure 2. Vanadium incoherent line measured at FLEXX using two different analyzers, PG and Heusler. The horizontal axis of the plot is A5, i.e. the analyzer rocking angle $\Omega$. The vertical axis represents detector counts per 5 seconds. The measurements were made in diffraction mode with $k_{i}=k_{f}=1.55 \AA^{-1}$, using an unpolarized incident beam. The offset in peak center positions is due to the different d-spacings of the Heusler $(1,1,1)$ and PG $(0,0,2)$ Bragg peaks.

PG analyzer for ideal reflectivities and ideal polarization. The full width half maximum of the peaks are $0.87^{\circ}$ for the PG analyzer and $0.73^{\circ}$ for the Heusler demonstrating the good alignment of the Heusler crystals.

Using the same vanadium rod, the horizontal focusing mechanics were driven to measure the optimize curvature value for each wavevector (Fig. 3, top). Figure 3 (bottom) shows the focusing gain as a function of wavevector. A gain factor of 3.5 for the flux at the detector for low wavevectors has been measured.

Straight through beam measurements were made with a fully polarized beam, a spin flipper was mounted in front of the Heusler analyzer in order to calculate the polarization of the whole experimental set up. Flipping ratios of $21.4(91 \%)$ were obtained. Better polarization ratios can be expected after optimizing the guide fields before, at and after the sample position. These values can be compared with polarization values at PANDA (FRMII) of about $92 \%$, where a Heusler monochromator and analyzer are used [9]. Full XYZ polarization analysis as well as the neutron resonance spin-echo [8] option can be requested when applying for beamtime at FLEXX.

\section{The MultiFLEXX multi-analyzer option}

Another major upgrade of the FLEXX spectrometer is the upcoming multi-analyzer option, MultiFLEXX, which combines the high sample position flux of a cold TAS with a multiplexing measurement of a broad range of $S(\mathbf{Q}, \omega)$ in one scattering plane - while retaining the full suite of sample environment capabilities on FLEXX.

Several multiplexed instruments or instrument options are available or are under construction at various neutron sources. The Flat-Cone diffractometer E2 at HZB [10] has been recently upgraded with an array of position sensitive detectors which can be tilted out of the horizontal plane.
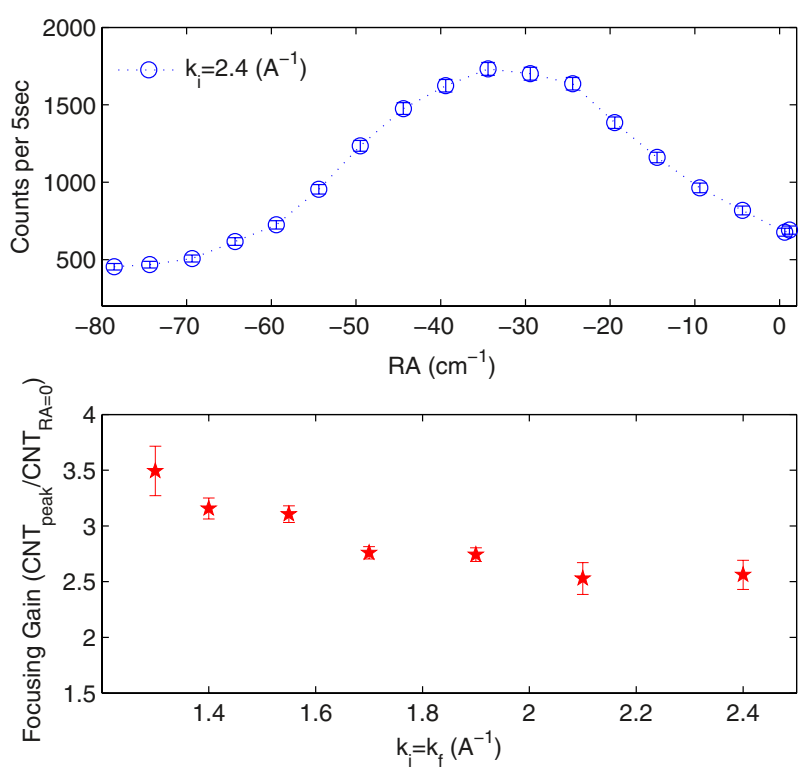

Figure 3. Top: intensity variation at the detector as a function of horizontal curvature RA. Bottom: focusing gain of the Heusler analyzer at different wavevectors. The gain is calculated from the detector counts at optimum focusing horizontal curvature RA normalized by the detector counts with flat analyzer $(\mathrm{RA}=0)$. The measurements were made with the incoherent line of a vanadium sample, using an unpolarized incident beam.

This instrument allows to detect elastic scattering over a continuous Q-range without energy analysis. The FlatCone multianalyzer setup at ILL [11] offers several discrete Q channels and energy analysis which can be chosen at either cold or thermal neutron wavevectors, $k_{F}=1.5 \AA^{-1}$ or $k_{F}=3 \AA^{-1}$. This option can be used at several three-axis spectrometers at ILL and is tiltable out of the scattering plane as well. A flexible multi-analyzer/detector option has recently become available at PUMA, FRM II, [12], which allows survey measurements in $(\mathrm{Q}, \omega)$. With this instrument option a single energy is associated with a particular Q channel while a large degree of flexibility for the positioning of the analyzer blades is provided. Related realizations are the Universal Focusing Option UFO available at IN12 at ILL [13] and the Re-Invented Triple-Axis spectrometer concept RITA realized at the SINQ Swiss neutron source of the PSI [14]. A Continuous Angle Multiple Energy Analysis (CAMEA) system is currently under construction at PSI. This multiplexed spectrometer back-end aims at detecting several final energies simultaneously over an extended range of wave vector transfers $Q$ similar to the MultiFLEXX option described here. The CAMEA concept is also proposed for an indirect geometry spectrometer for the future European Spallation Source ESS [15].

The design goal of the MultiFLEXX is to utilize the near-perfect neutron transmission of highly ordered pyrolytic graphite (HOPG) at low energies [16] to construct individual $2 \theta$-channels with several E-channels, by placing vertically scattering analyzer/detector sections behind each other in the neutron flight path [17] (see Fig. 4a). 

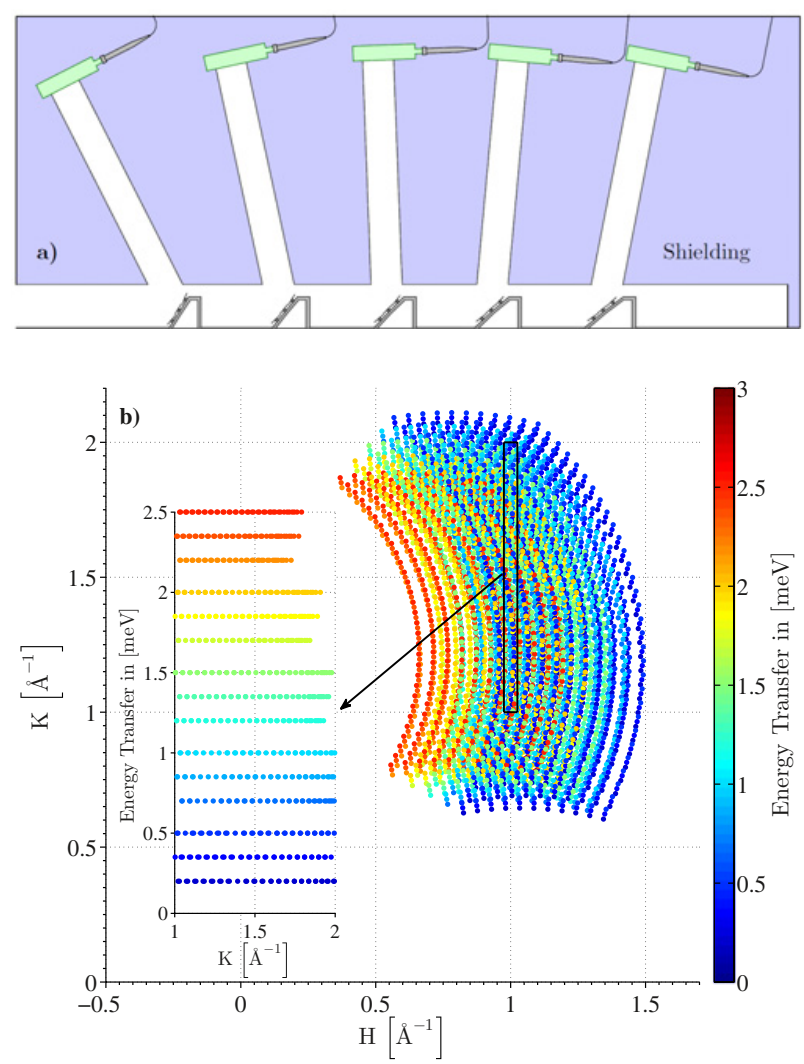

Figure 4. a) Cross-sectional view of a single $2 \theta$-channel. b) Q-range covered by a $\theta-\theta$ scan which probes the dispersion primarily along a particular direction in Q-space. For this illustration a cubic lattice has been assumed with $a=2 \pi \AA$. The data points correspond to 11 different $\theta$-values. For each of the $11 \theta$-values, 31 Q-values each at 5 different energy transfers are recorded. Finally, the scans are repeated for 3 different incident energies $E_{I}=5,4.83$ and $4.67 \mathrm{meV}$. In total this results in 5115 data points. The inset shows the energy transfer covered by those data points which fall into the marked rectangular region as a function wavevector for the direction of interest, here the crystallographic $K$-direction.

The particular case of the MultiFLEXX employs 5 fixed $E_{f}$-channels of $2.5,3,3.5,4$ and $4.5 \mathrm{meV}$. This invariably covers an energy-transfer range of $2 \mathrm{meV}$ which is fixed for this setup. The advantage of this simple approach to a multiplexing back-end is having no moving parts to control and calibrate - as well as being able to optimize the curvature of each analyzer for a single $E_{f}$. In order to optimize the MultiFLEXX for the limitations on out-of-plane solid angle coverage due to sample environment for the highest magnetic fields available, the out-of-plane angular coverage of the analyzers were chosen to be small. Hence, each analyzer consists of 3 vertically stacked plate-like $20 \times$ $20 \times 2 \mathrm{~mm}$ HOPG crystals (see Fig. 4a). For each channel we use a cylindrical He-3 tube-detector with a radius of $12.7 \mathrm{~mm}$ and an active length of $50 \mathrm{~mm}$ placed $400 \mathrm{~mm}$ away from the center of each analyzer in the out-ofplane Bragg condition. A total of $312 \theta$ channels for the MultiFLEXX will be built, covering an angular range of $77^{\circ}$ in $2 \theta$ with a gap of $2.5^{\circ}$ between two neighboring channels. The total solid angle covered by the MultiFLEXX is 10 times that of the standard FLEXX back-end. Given that the FLEXX front-end upgrade provided a factor of 5-10 in neutron flux - the MultiFLEXX has a gain factor of up to 100 as compared to the old FLEX, if a suitable broad $(\mathrm{Q}, \mathrm{E})$ range is under investigation in applied fields. For parametric studies targeted at a particular region in $(\mathrm{Q}, \mathrm{E})$ space, the MultiFLEXX underperforms the standard back-end of FLEXX, and hence it remains an option for the users.

The MultiFLEXX is designed for an incoming energy of $E_{i}>4.5 \mathrm{meV}$, where the energy resolution of the $E_{f}=2.5 \mathrm{meV}$ and the $E_{f}=4.5 \mathrm{meV}$ channels become comparable with $\triangle E_{F W H M}>0.09 \mathrm{meV}$. This is due to the fact that with a fixed set of low $E_{f}$ 's, the bandwidth of the incoming beam becomes more significant for the energy resolution as $E_{i}$ increases. Thus, for a chosen $(\mathrm{Q}$, E) range, one only has to perform the same Q-scan at three $E_{i}$ 's $0.15 \mathrm{meV}$ apart to have thoroughly covered an energy range of $2 \mathrm{meV}$.

Furthermore, one of the advantages of having the flexibility of a triple axis spectrometer back-end is that it offers a wide range of options regarding the choice of Qscan. One option is keeping the position of the back-end constant and merely performing a rotation of the sample, thus covering a constant length interval in Q. Another interesting option is performing a $\theta-\theta$-scan, i.e. rotating the scattering angle (A4) by the same amount $\theta$ as the sample rotation angle (A3) (see Fig. 4b). This type of scan allows for a more focused measurement in Q-space, making parametric studies more feasible. The scan shown in Fig. 4b) shows a total of only 33 settings. With a typical sample, MultiFLEXX could cover this volume in $(\mathrm{Q}, \mathrm{E})$ space in 24 hours, counting 45 minutes per setting.

In order to optimize the design of the $2 \theta$ channels and to investigate the performance of MultiFLEXX, we performed a range of ray-tracing simulations using the McStas Monte Carlo simulation package [18,19]. We used the same virtual front-end setup as in Ref. [4], proven to accurately simulate the real beam at the sample position. We simulated each energy channel separately at a fixed $2 \theta$ in $\mathrm{W}$-configuration. The width of the elastic line for each channel was simulated using a virtual vanadium sample.

As a measure of the inelastic resolution of the MultiFLEXX, we slightly modified the vanadium sample component to have the option of reducing the energy of the incoming neutron by a fixed amount corresponding to the channel in question. Thus, we simulated the performance in measuring a completely flat dispersion surface in $(\mathrm{Q}, \mathrm{E})$-space, a reasonable compromise between optimally focused and de-focused scenarios. The inelastic peak widths were investigated in the near-elastic regime using $E_{i}=5 \mathrm{meV}(\Delta E=0.5-2.5 \mathrm{meV})$, and in the intermediate energy regime by using $E_{i}=10 \mathrm{meV}$ $(\Delta E=5.5-7.5 \mathrm{meV})$. The flux on FLEXX exceeds 3 . $10^{7} \mathrm{n} / \mathrm{s} / \mathrm{cm}^{2}$ for $E_{i}<18 \mathrm{meV}$, but at these high energies the difference in $E_{f}$ will play a minor role and the resolution of the various channels will be coarse and similar. The energy widths and normalized intensity for each of the channels are given in Table 1. The energy resolution for all channels is comparable to the standard FLEXX resolution [4], due to the fact that the vertical 
Table 1. The sample-analyzer distances $\left(D_{\text {sample }}\right)$, the analyzer curvature $(\rho)$ and the simulation results for each channel. Three sets of simulations have been performed: (i) elastic scans $E_{i}=$ $E_{f}$, (ii) the incident neutron energy $E_{i}=5 \mathrm{meV}(\Delta E=0.5-$ $2.5 \mathrm{meV})$, and (iii) the incident $E_{i}=10 \mathrm{meV}(\Delta E=5.5-$ $7.5 \mathrm{meV})$.

\begin{tabular}{|c|c|c|c|c|c|}
\hline Channel & $2.5 \mathrm{meV}$ & $3 \mathrm{meV}$ & $3.5 \mathrm{meV}$ & $4 \mathrm{meV}$ & $4.5 \mathrm{meV}$ \\
$D_{\text {sample }}[\mathrm{mm}]$ & 1050 & 1220 & 1387 & 1552 & 1732 \\
\hline$\rho[1 / \mathrm{m}]$ & 0.76 & 0.6 & 0.5 & 0.41 & 0.37 \\
\hline$\Delta E^{\text {elas. }}[\mu \mathrm{eV}]$ & 53 & 78 & 103 & 130 & 157 \\
\hline$I^{\text {elas. }}[$ a.u. $]$ & 51 & 112 & 179 & 246 & 310 \\
\hline$\Delta E^{0.5-2.5}[\mu \mathrm{eV}]$ & 89 & 106 & 123 & 140 & 164 \\
\hline$I^{0.5-2.5}[$ a.u. $]$ & 264 & 326 & 358 & 369 & 372 \\
\hline$\Delta E^{5.5-7.5}[\mu \mathrm{eV}]$ & 260 & 265 & 274 & 283 & 293 \\
\hline$I^{5.5-7.5}[$ a.u. $]$ & 409 & 494 & 514 & 548 & 552 \\
\hline
\end{tabular}

angular coverage of the analyzers is rather small. It is interesting to note that the increased intensity from having a large resolution volume in the high $E_{f}$ channels $\left(V_{\text {res }}^{E_{f}} \propto\right.$ $\left.k_{f}^{3} / \tan \left(\theta_{A}\right)\right)$ is almost cancelled out by the decrease in the covered solid angle from having the high $E_{f}$ channels located further away from the sample position. However, in any case, both the analytical expression for the change in intensity due to the aforementioned effects and the experimental reflectivities of each analyzer need to be accounted for in the normalisation process.

\section{Conclusion}

The upgrade of the FLEXX primary spectrometer resulted in significant intensity gains across the accessible range of incident neutron energies. These gains allow spectroscopy on smaller single crystalline samples or likewise the use of intensity demanding options, such as polarized neutron modes (XYZ polarization analysis mode, NRSE mode) or the multiplexing back-end MultiFLEXX.

Polarized neutron work will significantly benefit from the recently commissioned Heusler analyzer. Careful characterization measurements have been performed and demonstrate optimum alignment of the individual Heusler crystals. Horizontal focusing, now also available for polarized neutrons, results in gain factors varying between 2.5 and 3.5. The experimental flipping ratio extracted from our very first measurements exceeds 20 and is sufficient for polarized neutron mode.

The new MultiFLEXX multi-analyzer option will simultaneously cover large regions in wavevector energy space bridging between TAS and TOF instruments. The MultiFLEXX option is thought to be particularly valuable in the presence of sample environment restricting the vertically accessible detector solid angle of standard TOF instruments. In conclusion, we have designed a very simple multiplexing option for FLEXX, with no moving parts and which is mountable in a day. We have simulated the performance of the MultiFLEXX and found the energy resolution comparable to the standard TAS resolution for all channels. A multiplexing back-end of this type offers a variety of different scan choices, and offers a unique option for performing parametric studies of a broad range in $S(Q, \omega)$.

FLEXX is offered in full user service and provides new capabilities for energy research, quantum magnetism, heavy-fermion systems and unconventional superconductivity. The upgrade and the availability of the new options puts the FLEXX triple axis spectrometer in a position where it can continue making leading edge inelastic neutron scattering measurements in fundamental material research.

\section{References}

[1] T. Krist, A.Tennant, Neutron News 25 4-7 (2014)

[2] P. Vorderwisch, S. Hautecler, F. Mezei, U. Stuhr, Physica B 213-214 866 (1995)

[3] M. D. Le, M. Skoulatos, D. L. Quintero-Castro, R. Toft-Petersen, F. Groitl, K. C. Rule, and K. Habicht, Neutron News 25 19-22 (2014)

[4] M. D. Le, et al. Nucl. Instrum. Methods Phys. Res. A 729 220-226 (2013)

[5] K. Habicht, M. Skoulatos, Journal of Physics: Conference Series 340012029 (2012)

[6] M. Skoulatos, K. Habicht, K. Lieutenant, Journal of Physics: Conference Series 340012019 (2012)

[7] M. Skoulatos, K. Habicht, Nucl. Instrum. Methods Phys. Res. A 647 100-106 (2011)

[8] F. Groitl, PhD thesis, TU Berlin (2013)

[9] E. Faulhaber, J. of Phys: Conference Series 211 012031 (2010)

[10] D. Hohlwein, A. Hoser, and W. Prandl, J. Appl Cryst. 19262 (1986)

[11] M. Kempa, B. Janousova, J. Saroun, P. Flores, M. Boehm, F. Demmel, J. Kulda, Physica B 385-386 1080 (2006)

[12] P. Link, G. Eckold, J. Neuhaus, Physica B 276-278 122 (2000)

[13] W. Schmidt, M. C. Rheinstädter, S. Raymond, M. Ohl, Physica B 350 e849 (2004)

[14] K. Lefmann, D.F. McMorrow, H.M. Rønnow, K. Nielsen, K.N. Clausen, B. Lake, G. Aeppli, Physica B 385-386 1083 (2006)

[15] P. G. Freeman, J. O. Birk, M. Markó, M. Bertelsen, J. Larsen, N. B. Christensen, K. Lefmann, J. Jacobsen, Ch. Niedermayer, F. Juranyi, H. M. Rønnow, arXiv:1406.5945 (2014)

[16] EPFL-REPORT-190504 - http://infoscience. epfl.ch/record/190504/files/PG Experimental_Results_1.pdf

[17] EPFL-REPORT-190509 - http://infoscience. epfl.ch/record/190509? ln=en

[18] K. Lefmann and K. Nielsen, Neutron News 10, 20, (1999)

[19] P. Willendrup, E. Farhi and K. Lefmann, Physica B, 350735 (2004) 